\title{
Are sex hormones promising candidates to explain sex disparities in the COVID-19 pandemic?
}

\author{
Maria Luisa Brandi ${ }^{1}$ iD
}

Accepted: 25 October 2021 / Published online: 10 November 2021

(c) The Author(s), under exclusive licence to Springer Science+Business Media, LLC, part of Springer Nature 2021

\begin{abstract}
Emerging evidence suggests that the novel Coronavirus disease-2019 (COVID-19) is deadlier for men than women both in China and in Europe. Male sex is a risk factor for COVID-19 mortality. The meccanisms underlying the reduced morbidity and lethality in women are currently unclear, even though hypotheses have been posed (Brandi and Giustina in Trends Endocrinol Metab. 31:918-27, 2020). This article aims to describe the role of sex hormones in sex- and gender-related fatality of COVID-19. We discuss the possibility that potential sex-specific mechanisms modulating the course of the disease include both the androgen- and the estrogen-response cascade. Sex hormones regulate the respiratory function, the innate and adaptive immune responses, the immunoaging, the cardiovascular system, and the entrance of the virus in the cells. Recommendations for the future government policies and for the management of COVID-19 patients should include a dimorphic approach for males and females. As the estrogen receptor signaling appears critical for protection in women, more studies are needed to translate the basic knowledge into clinical actions. Understanding the etiological bases of sexual dimorphism in COVID-19 could help develop more effective strategies in individual patients in both sexes, including designing a good vaccine.
\end{abstract}

Keywords COVID-19 $\cdot$ Estrogens $\cdot$ Androgens $\cdot$ Progestins $\cdot$ SERMs

$\begin{array}{ll}\text { Abbreviations } \\ \text { COVID-19 } & \text { Coronavirus disease 2019 } \\ \text { COPD } & \text { Chronic obstructive pulmonary disease } \\ \text { SARS } & \text { Severe acute respiratory syndrome } \\ \text { MERS } & \text { Middle East respiratory syndrome } \\ \text { ACE2 } & \text { Angiotensin converting enzyme type 2 } \\ \text { Ang } & \text { Angiotensin } \\ \text { TMPRSS2 } & \text { Transmembrane serine protease 2 } \\ \text { HSD3B1 } & \text { Hydroxy- } \delta \text {-5-steroid dehydrogenase, 3 } 3 \text { - and } \\ & \text { steroid- } \delta \text {-isomerase 1 } \\ \text { ARDS } & \text { Acute respiratory distress syndrome } \\ \text { SHBG } & \text { Sex hormone binding globulin } \\ \text { CVD } & \text { Cardiovascular diseases } \\ \text { MHT } & \text { Menopausal hormonal therapy } \\ \text { HSPAS } & \text { Heat shock proteinAS } \\ \text { SERM } & \text { Selective estrogen receptor modulator } \\ \text { CRP } & \text { C-reactive protein } \\ \text { ADAMs } & \text { A disintegrin and metalloproteinase } \\ \text { NLRP3 } & \text { Nod-like receptor pyrin domain 3 }\end{array}$

Maria Luisa Brandi

marialuisa@marialuisabrandi.it

1 Fondazione Italiana Per La Ricerca Sulle Malattie Dell'Osso, Florence, Italy

$\begin{array}{ll}\operatorname{ER} \alpha & \text { Estrogen receptor } \alpha \\ \operatorname{ER} \beta & \text { Estrogen receptor } \beta\end{array}$

\section{Sex-related differences in physiopathology}

There are several underlying differences in physiological functions between the two sexes and this fact implies a diverse incidence and severity of major diseases, as cardiovascular, musculoskeletal and immune disorders up to lifespan regulation [2-12]. As an example, $80 \%$ of patients with autoimmune diseases are females and similarly $80 \%$ of patients with osteoporosis are women [13-15]. Conversely, cardiovascular diseases are anticipated of at least a decade in men when compared to women [16]. In general, men have a lower life expectancy than women, but women spend their extra years with higher levels of illness, the so called gender health paradox [17].

Comorbidities have been repeatedly reported as key clinical prognostic factors in COVID-19 infection and a sex-related dimorphic phenotypical expression of the main diseases underlying the risk for COVID-19 complications could explain the different outcomes between sexes in 
this pandemic. Main disorders to consider are hypertension, type 2 diabetes and obesity, ischemic heart disease, chronic obstructive pulmonary disease (COPD), and renal insufficiency.

However, until recently very little attention was given to gender differences in the physiopathology of major human organs and systems, with the exception of the reproductive apparatus.

Potential causes of sex-based differences in normal physiology and disease can be recognized in biological (genetic and hormonal) factors, while gender differences are motivated by psicosocietal-cultural factors (i.e. excessive alcohol consumption, drug use and smoking being excessive in males). Moreover, awareness of a given disorder may be different in females and males, as it happens in osteoporosis, prevalently diagnosed and cured in females [18].

The obvious genetic differences between males and females lie in the fact that females have two $\mathrm{X}$ chromosomes, whereas males have a $\mathrm{Y}$ and a $\mathrm{X}$ chromosome. From an endocrine point of view sex hormones influence physiology in both sexes with changing roles during human life, from childhood to adolescence to adulthood and to senescence. Finally, societal factors, such as lifestyle and daily environment, can play a role in the dimorphic expression and morbidity of human diseases in the two sexes..

The assumption that there are minimal differences in the pathophysiology on men and women had as a natural consequence the lack of controlled clinical trials carried out in the two sexes and analyzed separately. Increasing evidence has shown that not only the selection of a given compound and the therapeutical effectiveness of any pharmacological interventions vary between the two sexes, but also that sex differences exist in adverse drug events [19, 20]. Considering that we are in the era of personalized medicine sex and gender cannot be ignored any longer.

The recent described sex- and gender-related disparities in morbidity and mortality in COVID-19 infection opened to a renewed interest on the sexual dimorphism in viral infection that affect the respiratory system [1,21].Finding the pathogenetic mechanisms that underlie these differences could help develop more effective prevention and treatment strategies for individual patients and create equitable policies.

The first element to be considered in the future is developing sex targeted therapies. The information among multiple pharmacological options in COVID-19 evidences different efficacy and safety results, but data on sex-related responses are currently limited. Neither sex disaggregated data on benefits, adverse events and long-term safety on vaccination are available. In addition, the prevalent inclusion of men in randomized clinical trials with future extrapolation of results to women should be addressed.

\section{Epidemiological evidences}

The COVID-19 pandemia is likely to have started in China and rapidly Europe, the United States, and South America were affected (https://Coronavirus.jhu.edu/map.htlm). While men and women have the same prevalence of the disease, sex differences emerged in terms of case fatality (deaths/reported cases) evidenced that the male/female ratio is always above 1.1 in 34 out of the 35 countries that provided sex disaggregated data (only for Pakistan, the ratio is 0.9 ), independently of age [22]. Men account for over $50 \%$ of total deaths and almost twice as many men with COVID-19 suffer severe symptoms or death in comparison to women $[23,24]$.

In addition to sex dimorphism in fatality of the disease, epidemiological studies have shown an age-dependent susceptibility, with older individuals experiencing, along with a decline in sex hormone production, also a more severe COVID-19 illness.

To sum up, currently available studies, suggest that both young people and women are less susceptible to severe COVID-19 infection outcomes, regardless for their geographical origin.

Altogether, these data point to a potential role of sex hormones, not just in the predisposition to COVID-19 infection, but in the clinical course of the disease.

Similar observations were already reported for other coronavirus epidemics. Previous studies in patients affected by the severe acute respiratory syndrome (SARS) and Middle East respiratory syndrome (MERS) epidemics evidenced a similar age-dependent susceptibility and sex-related diversities in both morbidity and fatality [25-28].

Sexual dimorphism in COVID-19 should not come as a surprise because it is known that men and women respond to viral infection differently $[29,30]$.

Policies should take care also of gender differences related to social and economical diversities in the two sexes. Women represent the prevalent health workforce, being thereby at higher risk of infection. Women home workload increased with the closure of the schools and this will limit their work and economic opportunities. Women are still not included in decision making for outbreak response, while their inputs would be important for the society. While the fragility of male patients affected by COVID-19 is clearly in the agenda of the decisors, the direct and indirect consequences of the pandemia in women lifes risk to perpetuate gender and health inequities.

Although the mortality rate from COVID-19 appears higher amongst men, potentially as a result of both biological and social factors, the recession that will follow the pandemia could have a gendered impact on women in terms of morbidity and mortality [22]..Governments and global 
health institutions are advised to consider the sex and gender effects of the COVID-19 outbreak, both direct and indirect, and conduct analyses of the gendered impacts of the multiple outbreaks. This will make possible to mitigate the impact of the pandemic on long-term health inequalities amongst women and men.

\section{Pathogenesis of gender- and sex-related disparities}

The knowledge of the pathogenetic mechanisms that underlie COVID-19 will make possible to understand who is most at risk of severe outcomes of the disease and how to adjust therapeutic interventions accordingly. Despite this striking evidence for COVID-19 infection, limited studies consider different therapeutic approaches for the two sexes. In a scenario where no specific antiviral drugs have been developed to treat COVID-19 infection and control disease evolution, a better understanding of the pathogenetic bases for sex-linked dimorphism for this disease should contribute to characterize new therapeutical targets.

The different vulnerability of males and females to COVID-19 infection is an evidence that cannot be discussed, even though an explanation for this is not available. Beside a different hormonal milieu, there are other potential mechanisms that may explain why women are less prone to severe COVID-19 disease. Scientists are offering some clues to understand the basis for this disparity.

\subsection{Gender-related causes}

There are many ways that gender can impact on COVID-19 statistics. Attempt to explain the higher morbidity in men included social differences, smoking, social behaviour, and delay in medical care between sexes [31]. This echological interpretation can be eclipsed by individual genetic, immunologic, and hormonal interferences. Notably, these factors will all increase susceptibility to COVID-19, but they cannot explain the findings of sex differences.

In the process of recognizing possible factors contributing to gender-and sex-based discrepancies, since age is also a risk factor for COVID-19 severity and mortality, comparisons of the COVID-19 outcomes in both sexes should be performed after age matching.

\subsection{Genetics}

Genetic differences between males and females are well recognized. An important difference is the dosage of the sex chromosomes, that are the basis not only of sexual determination, but also of qualitative ( $\mathrm{Y}$ versus $\mathrm{X}$ chromosome) and quantitative (one or two $\mathrm{X}$ chromosomes) expression of functional genes. While in males the $\mathrm{Y}$ chromosome is smaller than the $\mathrm{X}$ chromosome and contains mainly genes coding for sex-specific effects and testis development, in females the second $\mathrm{X}$ chromosome contains a number of genes, that if excluded from inactivation, may contribute to the greater longevity of females relative to males [12]. This interpretation is supported by the fact that male birds, that utilize the $\mathrm{ZW}$ sex determination system (males $\mathrm{ZZ}$ and females $\mathrm{ZW}$ ), have a longer lifespan than females.

Notably, multiple genes important in the innate and adaptive immune response to viral infections sit on the $\mathrm{X}$ chromosome [32, 33]. In females, who bear two X chromosomes, a process known as $\mathrm{X}$ chromosome inactivation prevents the over-expression of X-linked genes, but some genes, including those controlling immune function, can escape this process. As a consequence genes can double their expression, with expected functional consequences.

Another important gene that sits on chromosome $\mathrm{X}$ region p22 and can be over-expressed in females is the gene encoding the receptor mainly responsible for SARS-Cov-2 cellular entry, the angiotensin converting enzyme type 2 (ACE2). The $A C E 2$ gene encodes a type I carboxypeptidase glycoprotein of about $100 \mathrm{kDa}$, composed by 805 amino acids and located as an ectoenzyme on the surface of the cells. ACE2 enzyme acts exclusively as a carboxypeptidase removing a C-terminal amino acid from Angiotensin II (Ang II) generating Ang(1-7) or from Ang I forming Ang(1-9). The Ang(17) acts through its G-protein coupled receptor Mas [34]. The protein is expressed on the surface of lung epithelial cells and other tissues, that regulate the renin-angiotensinaldosterone system [35]. The ACE2 is linked to a reduced risk of developing hypertension, cardiovascular diseases and it is also protective in the lungs against edema, permeability, and pulmonary damage [35]. These features point to the control of the expression of the ACE2 gene as a therapeutic strategy in hypertension and heart failure. The $A C E 2$ gene is regulated at multiple levels including transcriptional, posttranscriptional and post-translational through its shedding from the cell surface. The lung expression of the ACE2 gene decreases with age, prevalently in men [36]. This, together with a potential double dose of $A C E 2$ gene in women, could explain the higher COVID-19 lung morbidity and mortality in elderly men. Also the mechanism of acute myocardial injury caused by SARS-CoV-2 during severe COVID19 disease might be related to the inhibition of the ACE2 catalytic activity [37]. Humans are not equal with respect to the expression levels of the cellular ACE2 enzyme and $A C E 2$ gene expression and/or polymorphisms could influence both the susceptibility of people to SARS-CoV-2 infection and the outcome of the COVID-19 disease [35, 38]. Further exploration of the relationships between the virus 
and the ACE2 gene expression and genotype should help to better understand the pathophysiology of COVID-19.

Genetic factors may also influence the geographical spread of COVID-19. An example is offered by the hydroxy$\delta$-5-steroid dehydrogenase, $3 \beta$ - and steroid $\delta$-isomerase 1 $(H S D 3 B 1)$ gene, that is located at $1 \mathrm{p} 12$ and whose encoded protein is involved in the periferal synthesis of potent androgens from adrenal precursors. Notably, the most frequent hyperactive $H S D 3 B 1125 \mathrm{C}$ adrenal permissive allele is found in the Italian and Spanish populations [39].

Another androgen-related gene is the transmembrane serine protease 2 (TMPRSS2) gene, that sits in chromosome 21 region 21q-22.3 and encodes the transmembrane serine protease 2 capable to facilitate entry of viruses, including SARS-CoV-2 into host cells. This serine protease cleaves the spike protein for viral priming. SARS-CoV-2 entry into the cell depends on two factors, the ACE2 receptor and the cellular protease TMPRSS2, not surprisingly co-expressed in tissues targeted by the virus. From a genetic point of view, the rs 12329760 TMPRSS2 gene polymorphism could increase the chance of faulty expression of the gene itself [40], while the e QTL variant rs35074065, highly represented in the Italian population [41], is linked with overexpression of the TMPRSS2 gene and this may give rise to enhanced susceptibility to viral infection in Italians.

Bilateral pneumonia were observed in males with androgenic alopecia, a sign of hyperandrogenism, and this latter condition is also associated with short CAG repeat length of the gene encoding the androgen receptor [42]. It is hypothesized that worse outcomes of COVID-19 in men may be associated with CAG repeat length on the gene encoding for the androgen receptor.

Analyzing the distribution of gene polymorphisms could be helpful to understand the different susceptibility to COVID-19 outcomes. In a recent paper genome-wide association analysis made possible to uncover genes that segregate with mortality for COVID-19, prevalently related to host viral defense mechanisms and mediators of inflammatory organ damage [43].

Genetics play an important role in COVID-19 outcomes and sex hormones are important mediators of this genetic regulation.

\subsection{Immunological response}

Evidences point to profound differences between males and females in the immunological response to viral infections, with a diverse propensity also to autoimmunity [29]. Sex disparities in prevalence, intensity, and outcome of viral infections are conserved in evolution from the Drosophila melanogaster up to humans $[44,45]$. These differences can easily explain diversities in incidence, duration, severity, and fatality rates following a viral infection.

In dealing with viral infections, female immune system acts differently than in males, developing a stronger immune response leading to viral clearance. In general, antibody production level is higher in women when compared to men and lasts longer [46]. Notably, the general morbidity from seasonal influenza are consistently higher in men than women at all ages [47].

While our understanding of the exact immunological mechanisms underlying these differences is still in its infancy, the published data support existing sex-related diversity in the composition, epigenetic regulation, and function of immune cell populations [48-51].

Sexual dimorphism is evidenced also in coronvirusesrelated inflammatory response, that results from a dysregulated response of the immune system [52, 53]. Indeed, elevated IL-6, C-reactive protein (CRP), and excess in cytokines release segregate with the acute respiratory distress syndrome (ARDS) and are higher in males than in females. The fact that the stronger immune response to viral infections in women segregate with a more modest inflammatory response to COVID-19 is paradoxical. An explanation for this surprising behaviour is that the hyperinflammatory response seen in COVID-19 male patients represents the natural consequence of the ineffective early antiviral immunity typical of men [54].

Interestingly, examining SARS-Cov-2-specific antibodies, plasma cytokines and blood cell phenotype in male and female COVID-19 patients, men have higher plasma levels of innate immune cytokines and women mounted significantly more robust $\mathrm{T}$ cell activation [55].

\subsection{Cardiovascular comorbidity}

It has become clear that cardiovascular co-morbidities are more prevalent in males and patients with cardiovascular disease infected with COVID-19 have a worse prognosis [54]. In view of the fact that the biological sex plays an important role in the immune response and that inflammatory cytokines have been implicated both in COVID-19 and in cardiovascular dysfunction, it has been suggested that the sexual dimorphism in the immune reaction may influence the cardiovascular diseases and COVID-19 interactions [56].

Notably, cardiovascular co-morbidities associated with COVID-19 encompass arterial hypertension, ischemic heart disease, and atrial fibrillation, all conditions characterized by an abnormal ACE/ACE2 ratio in humans and in animals [57]. Moreover down-regulation of ACE2 may favor endothelial dysfunction and coagulopathy [58].

Further excavating into the pathogenetic bases of the interactions between cardiovascular disease, COVID-19, and 
the inflammatory response, it was shown that the adaptive immune response in SARS-Cov-2 infection declines more rapidly in hyperlipidemia [59].

Obesity also demonstrates sexual dimorphism and it has been associated with COVID-19 severity, death and inflammation [60, 61].

Initial studies found increased severity of COVID-19 in patients with diabetes mellitus, with consequent hyperglycemia that may modulate immune and inflammatory responses [62]. The main recommendations for COVID-19 patients with diabetes mellitus should strictly follow general preventive rules, with frequent monitoring of glucose levels, engagement in physical activity, healthily eating, and control of concomitant risk factors in both sexes [63]. Notably, type 2 diabetes mellitus is more frequently diagnosed at younger age and at lower body mass index in men; however, the most prominent risk factor, which is obesity, is more common in women [64]. In addition, diabetic women have greater increases of cardiovascular mortality, myocardial infarction, and stroke mortality than men, compared with non diabetic subjects [65].

Overall, clinical and experimental evidence support the protective actions of endogenous estrogens towards diabetes susceptibility [66].

\subsection{Lung physiopathology}

In COVID-19 lungs become the door of entrance to the virus and often the cause of death for the affected patient. The ARDS is a major cause of mortality in COVID-19, developing as a complication of pneumonia with treatment being limited to supportive interventions. The understanding of the pathogenesis of this disorder and of the mechanisms that control its evolution becomes a very important area of investigation. Can sex-related differences play a significant role?

Sex has a significant effect on respiratory system structure and function. These differences may by driven by sex hormones and environmental exposures and may result in an increased risk for certain lung diseases, including COVID19 , as well as differences in outcomes.

Sex biological differences in lungs start from the human lung development, manifesting as early as at 16 weeks of gestation and can persist throughout fetal maturation. For example, more mature lung phenotype has been described in the female fetus than in the male fetus [67]. It is well known that the synthesis of surfactant in the fetal lung is sexually dimorphic [68]. This can explain the disadvantage that male lungs have at birth.

Women have proportionally smaller lungs and airways than men and men and women's rib cages differ in terms of shape [69]. Males have more bronchioles, fewer alveoli per unit area, a decreased expiratory flow rates (for increased smooth muscle and thicker airway walls) and smaller central airways. These phenotypic differences affect pulmonary function at rest and during exercise. Another potential sexrelated difference can be found in the damage exerted by smoking on the respiratory function, with females reporting more symptoms than males with similar smoking burden [70].

Fragility vertebral fractures represent another potential difference between the two sexes, as these influence the respiratory function and prognosis of affected patients [71] and are more frequently diagnosed in females [18].

The impact of these sex-related differences on disease susceptibility and progression, particularly for the interstitial lung disease, remain incompletely elucidated [72]. While much still needs to be learned about the mechanisms responsible for sex differences in lung diseases, including COVID19, future cellular and clinical studies might shed light on potential mechanisms responsible for these differences in the hope to unveiling potential targets for intervention.

\subsection{Coagulation}

COVID-19 physiopathology is not only characterized by an exacerbated inflammatory response, but also by a hypercoagulable state. The risk of thromboembolism is higher in men throughout the life cycle, while women exhibit a lower risk than men in their fertile years, gradually increasing during menopause [73].

One would be tempted to use estrogens to prevent thromboembolism in COVID-19. However, in conditions of supraphysiological estrogen levels, such as pregnancy, we witness an increase in the coagulation function.

\subsection{Gonadal function}

The clinical relevance of sex hormones' signaling in diseases' pathogenesis lies in the variations of severity and prognosis in relation with levels and fluctuations of circulating hormones during puberty, menstrual cycle, pregnancy, menopause, and aging.

\subsubsection{Males}

During the ageing process, there is a progressive decline in serum total and free testosterone levels, increase in sex hormone binding globulin (SHBG), and moderate increase in luteinizing hormone $(\mathrm{LH})$. Incidence of hypogonadism (total testosterone $<3 \mathrm{ng} / \mathrm{ml}$ ) accounts $15-20 \%$ in men between 49 and 75 years of age [74].

Low serum levels of testosterone may trigger a detrimental cytokine dysfunction and are associated with aging and obesity and other chronic diseases, leading to systemic inflammation, endothelial dysfunction and increased platelet activity, predisposing to thrombosis and CVD. 
Consequently, men with lower levels of testosterone are more prone to develop pulmonary and cardiac complications in COVID-19.

On the other side testosterone predispose men to a less effective immune response against infectious agents.

Testosterone levels behave as a true Bifront Janus towards the outcomes of COVID-19.

Antiandrogens are used routinely or evaluated in clinical studies for the treatment of both prostate and mammary cancer and are well tolerated in men and women. The use of these compounds leading to downregulation of TMPRSS 2 gene expression might reduce the susceptibility to COVID-19, as it emerges from clinical data [75]. Also proxalutamide, a potent second generation non steroidal androgen receptor antagonist, seems to reduce in males by $91 \%$ the hospitalization rate in a Brazilian population [76]. This study was criticized and future ongoing trials will try to offer a realistic view of the problem. Other findings support a role of the antiandrogenic therapy in the natural history of COVID-19, as androgen-deprivation therapy for prostate cancer appear to reduce the risk of infection by SARS-CoV-2 [77].

Cancer patients have been reported to be at higher risk of COVID-19 complications and deaths [78]. For all these reasons in prostate cancer the use of androgen deprivation therapy and antiandrogens are certainly indicated both in COVID-19 and in non-infected patients.

\subsubsection{Females}

The variability in circulating estrogens in women presents a diversification certainly higher than what seen in males, as exemplified by mestrual cycles, pregnancy, and menopause.

Current literature does not include pregnant women as an a priori risk group for COVID-19, even if COVID-19 infection in pregnant women creates additional challenges for various reasons [79]. The increase in estradiol and progesterone in pregnancy could contribute to a hormonal milieu which shifts the cytokines signature towards an anti-inflammatory state. Future studies will have to address COVID-19 mortality during pregnancy compared to early postpartum in order to determine whether the hormonal environment of the third trimester, in particular progesterone, is protective [80].

In postmenopausal women estradiol sharply declines with concomitant reduction in total and free testosterone and SHBG. The most represented estrogenic hormone is estrone, with high levels of circulating gonadotrophins. Interestingly ACE2 has been described also in the granulosa cells and its expression is increased with the rise in LH [81].

Factors such as obesity and chronic liver disease can also affect the concentrations of estrogens amongst individuals [82]. In a recent study it was demonstrated that mortality was higher in postmenopausal women than in premenopausal women, further supporting the protective role of female sex hormones [83].

A special attention should be posed towards men and women under hormonal therapy for prostate and mammary cancer, a therapy that most physician preferred during the pandemic to allow safe deferral of surgery [84].

Altogether these findings open to the hypothesis that the ageing-related decrease in testosterone levels may contribute to the progression of the disease in males. Conversely, in postmenopausal women, either the presence of estrone and high gonadotrophin levels may exert a protective effect.

\section{The role of sex hormones}

Overall, results of studies in various animal species, including Drosophila, are consistent with the conclusion that hormonal signaling in general, and steroid hormone signaling in particular, are mechanisms that control life span across species, most likely through regulation of trade-offs between reproductive metabolism versus mitochondrial and somatic maintenance [12]. There is speculation that sex steroid hormones may contribute to the sex-related disparities in fatality from COVID-19. The weight of sex hormones' action should encompass the natural waning of hormone production over the course of one's lifetime, a variable that accounts for the increased risk of the overall elderly population [85].

The different concentrations of estrogen, progesterone and androgen between females and males are likely to influence COVID-19 outcomes. This is important for two main reasons, first because COVID-19 may alter, even if only transiently [86], the function of the hypothalamic-pituitary gonadal axis, decreasing the secretion of gonadal hormones and also because gonadal hormones are amenable to therapeutic intervention.

\subsection{Estrogen}

Estrogen and the response to estrogen [through the estrogen receptor $\alpha(\mathrm{ER} \alpha)$, the estrogen receptor $\beta(\mathrm{ER} \beta)$ and the $\mathrm{G}$ protein-coupled receptor] could have an impact on the outcomes of COVID-19.

The correlation between sex hormones, disease severity, and death rates reveals the potential of utilizing hormone replacement therapy to modulate the immune response to increase resilience to adverse disease outcomes [87, 88]. Hormonal therapy may be possibly proposed as a method of boosting immunity in individuals who are most at risk of fatality by COVID-19 and to prove this hypothesis shortterm clinical trials are ongoing in both men and women ([23], Table 1). 
Table 1 Sex hormones-targeted trials from the World Health

Organization database, the EU Clinical Trials Register and the clinicaltrials.gov

\begin{tabular}{llll}
\hline STUDY CODE & DRUG & POPULATION & OUTCOMES \\
\hline NTC04359329 & Estradiol patch & Men and women & COVID-19 \\
& Progesterone oral & COVID-19 affected & complications \\
NTC 04865029 & Estradiol patch & Men and women & COVID-19 \\
& Progesterone oral & COVID-19 affected & symptoms \\
CTRI/2020/09/ 027622 & Estradiol oral & Men and women & COVID-19 \\
EUDRACT:2020-003403-33 & Esterol oral & COVID-19 affected & progression \\
& & Men and women & COVID-19 \\
EUDRACT:2021-000320-35 & Bazedoxifene & COVID-19 affected & progression \\
& & Men and women & COVID-19 \\
NTC 04365127 & Progesterone sc & COVID-19 affected & progression \\
& & Men & COVID-19 \\
NTC 04374279 & Enzalutamide oral & Men and women & COVplications \\
& & COVID-19 affected & worsening \\
NTC 04475601 & Enzalutamide oral & Men and women & COVID-19 \\
& & COVID-19 affected & progression \\
NTC 04509999 & Bicalutamide oral & Men & COVID-19 \\
NTC 04446429 & & COVID-19 affected & progression \\
& & Men & COVID-19 \\
& & COVID-19 affected & hospitalization \\
\hline & & &
\end{tabular}

The positive actions of estrogens in cardiovascular physiology are recognized in the anti-inflammatory effect, in the dilatation of the arterial wall, in the endothelial function, and in the intravascular coagulation [1]. Among the mechanisms proposed to mediate sex-specific differences in cardiovascular disease, signaling via the ACE2/ Ang(1-7)/Mas axis represents a promising candidate pathway by which female sex and/or estrogen may be protective from incidence and severity of ARDS [89]. The estradiol-induced increase in the expression of ACE2 and Ang1-7 opens to the possibility of counteracting the deleterious effects of Ang II in COVID-19 [90].

Estradiol is also able to inhibit the production of the TMPRSS2 protein, necessary for trimming and activating the SARS-CoV-2 spike protein to bind ACE2 [91] and to increase the expression of A Disintegrin And Metalloproteinase (ADAMs) mainly ADAM-17, which is able to ckeave the ACE2 ectodomain with release of high soluble circulating and SARS-CoV-2 neutralizing ACE2 [92].

Endogenous estrogen exert also a protective action towards type 2 diabetes mellitus through the ER $\alpha$ activation in various tissues, including the pancreatic beta cells, but also the role of sex chromosomes, fetal/neonatal programming, and epigenetic modifications need to be further investigated in the control of diabetes mellitus [66].

The data published demonstrate that estrogens have profound effects on respiratory physiology, but the results are limited to animal and not human studies. In mice estrogen therapy inhibits the activation of the nod-like receptor pyrin domain 3 (NLRP3) inflammatory and therefore it ameliorates airway inflammation and hyper-responsiveness [93].

A direct inhibitory effect of estradiol on SARS-CoV-2 infection was also demonstrated in vitro using the VERO E6 cells [94]. Moreover, the ER $\alpha$ is transcriptionally associated with ACE2 and TMPRSS2 genes' expression in the human atrium, revealing for the first time a functional modulators mechanism of ERs for the structural elements that control SARS-CoV-2 entry into the host cells.

The modulation of the estrogen pathway as a therapeutic strategy for COVID-19 is at the moment under evaluation in ongoing clinical trials ([95] and Table 1).

\subsection{Progesterone}

Progesterone, the second major female sex-steroid hormone, with major actions on the reproductive system, but also with critical roles in other tissue systems, such as the mammary gland, the cardiovascular system, the neurodevelopmental process in the central nervous system, and the bones. In normal healthy males the levels of progesterone are considerable, with a decline with age [96].

Studies on the role of progesterone in COVID-19 burden are limited, even though evidences exist.

Examples of this influence are the known antiviral effects of progesterone against HIV and the influenza virus, the immunomodulatory function of high doses of progesterone during pregnancy and the decrease of progesterone circulating levels in SARS patients [23]. 
This is the reason for the recent development of clinical trials to demonstrate progesterone-related anti-SARS-CoV-2 effects ([23] and Table 1).

\subsection{Androgen}

It has been reported that ACE2 is a constitutive product of Leydig cells, thus suggesting a potential role of SARSCoV-2 in controlling testosterone secretion [97].

Androgens act as bidirectional regulators of COVID-19 severity, as the majority of males with COVID-19 exhibit testosterone deficiency. Indeed, the association between an increase of proinflammatory state and a decline of testosterone levels is often observed, either in the ageing man or in the hypogonadal disorders [98]. Altogether these data suggest the use of androgens as adjuvant therapy for patients with COVID-19. However, the collected information do not go in the same direction, as androgen appear to stimulate the expression of molecules, that facilitate the SARS-CoV-2 infection.

Notably, androgen are known to promote airway branching, but less surfactant [99]. Interestingly, the gene encoding the TMPRSS2 enzyme is regulated by androgens in a lung-derived cell model [100], but it is not known if this protease is regulated by androgen in physiological settings [101]. In the case of a positive answer, inhibition of the androgen response could suppress the TMPRSS 2 enzyme and, therefore, the viral entry into the cells. Testosterone, a known biomarker of prostate cancer when fused with ERG [102], may favor the progression of COVID-19 through the activation via the androgen receptor of the transcription of the TMPRSS2 gene [102].

Androgen can cause severe infection in men by affecting the immune response through the increase of cytokines production and reducing the antibody response to the infectious conditions [103]. This issue becomes very important as the cytokines storm is seen in patients with severe COVID-19. Severity of lung involvement during infection with COVID19 is correlated with hyperandrogenism, especially in the elderly patients [104].

The antiandrogen enzalutamide downregulates the expression of the TMPRSS 2 gene and reduces cellular entry on SARS-CoV-2 in human prostate and lung cells [105].

Overall these findings justify the severity of the COVID19 reported in males and open to the possibility of an antiandrogenic therapy in the hyperandrogenic phenotype. Indeed, androgen-modulating drugs have bene proposed as a potential treatment for COVID-19 [106] and prostate cancer patients receiving androgen deprivation and anti-androgens therapy appear to be partially protected from SARS-CoV-2 infection [107].

Clinical trials are ongoing to evaluable the role of antiandrogens in COVID-19 affected males (Table 1).

\subsection{Pituitary-gonadal axis}

Information available on the pituitary-female gonadal axis in COVID-19 is limited. As ACE2 is expressed in the ovary and in the endometrium, it can be speculated that these tissues might be the targets of the SARS-CoV-2 [97, 108, 109].

Similarly, human testes-primarily spermatogonia, Leydig and Sertoli cells-express both high levels of ACE2 and TMPRSS2 genes [110]. This opens to the possibility of an interference of SARS-CoV-2 with testosterone secretion and sperm production. The increased secretion of $\mathrm{LH}$ in SARS-CoV-2 infected males with a reduced testosteroneto- $\mathrm{LH}$ ratio points to a direct testicular damage of the testis in COVID-19 [111].

\section{Compounds interfering with the response to sex hormones}

\subsection{Menopausal hormone therapy}

For menopausal hormone therapy (MHT) both synthetic estrogens and natural estrogens (i.e. estradiol and conjugated equine estrogens) were introduced and all designated simply as exogenous estrogen without a real distinction. This apparently unrecognized heterogeneity, together with the combination with synthetic or natural progestogens resulted in a chaos regarding effectiveness and safety of MHT.

This confusion will translate in a great difficulty in promoting the use of estrogen in the prevention or therapy of COVID-19 in postmenopausal women or even in men. If a suggestion could be made, this should be based on the recognized anti-inflammatory and immunomodulatory effects of natural estrogens in health women. Indeed, in a recent large retrospective study in postmenopausal women affected by COVID-19 in treatment with estradiol the fatality risk is reduced more than $50 \%$ [112].

Cessation of MHT should not be recommended in women with mild or moderate COVID-19, giving the preference to transdermal applications of estradiol [113].

\subsection{Contraceptives}

Estrogen containing contraceptives increase the production of coagulation factors, while decreasing the production of anticoagulant factors. An 80 year period of synthetic estrogen use as contraceptives induced a fear of even endogenous estrogen.

Oral contraceptives were developed in the early 60's, comprising predominantly the synthetic estrogenic compound ethinylestradiol, soon linked to risk of venous thromboembolism, stroke and miocardial infarct. Ethinylestradiol cannot be assimilated to natural estrogen, as it acts not as a 
natural binder but it deregulates the ERs function, leading to toxic complications and increased risk of ER negative mammary cancers [114].

Combined hormone contraception should be discontinued in COVID-19 patients admitted to the hospital and in less serious conditions or in the case the therapy is needed for reasons other than contraception the treatment should be switched to progestogen-only contraceptives [113].

\subsection{Phytoestrogens}

Several commercially available herbal medications are already being used as prophylactic agents against respiratory distress occurring due to flu and common cold.

Interestingly, natural plant phytoestrogens, able to interact preferentially with the ER $\beta$, can reduce pro-inflammatory cytokines and also alleviate and treating disseminated intravascular coagulation $[115,116]$.

Different natural products contain plenty of phytoestrogens that can compete with the viral spike recognition also through their interaction with the substrate binding domain $\beta$ of Heat Shock Protein AS (HSPAS), a recognition site for SARS-CoV-2 [117]. Interestingly HSPAS is recognized also by sex hormones, as estrogen, progesterone and testosterone, with phytoestrogens and estrogen showing the the highest affinity binding.

Phytoestrogens can represent an attractive possibility for COVID-19 patients and clinical studies are undergoing to demonstrate the efficacy of these natural products in ameliorating the outcome of the disease.

\subsection{Selective estrogen receptor modulators}

Tamoxifen, a major treatment strategy in mammary cancer patients, is the first Selective Estrogen Receptor Modulator (SERM) evaluated for its potential role in COVID-19 and this for several reasons. First, tamoxifen binds to the androgen receptor restraining directly its signaling and leading to TMPRSS2 downregulation [118, 119]. Moreover, tamoxifen independently of the estrogen receptor increases endolysosomal $\mathrm{pH}$, even more than the antimalarian chloroquine, thereby potentially inhibiting SARS-CoV-2 entry [120].

In early 2020 Brabek et al. were the first to propose the SERM bazedoxifene as a promising candidate to inhibit the IL6/cytokine storm [121]. Soon after the SERM raloxifene was identified as a molecule of great interest for potential effectiveness against SARS-CoV-2 through the Excalate$4 \mathrm{CoV}$ supercomputing platform (a tool used for repurposing old drugs for new indications) based on antiviral targeting. Interestingly raloxifene, as phytoestrogens, binds preferentially to ER $\beta$.
Both drugs were developed for the treatment of osteoporosis and both prevent the binding of IL6 to its receptor [122]. Moreover, the two SERMs could be effective against SARS-CoV-2 entry and replication, as shown in in vitro studies [123].

SERM molecules have the advantage to be beneficial to both male and female patients, without any major side effect.

Clinical studies are ongoing to support the potential use of bazedoxifene and soon raloxifene to prevent COVID-19 progression (Table 1).

\subsection{Xenoestrogens}

Environmental stressors have already been suggested to contribute to the severity of the disease, opening the avenue to compare the biological pathways triggered by environmental stressors with those involved in COVID-19 severity.

Xenoestrogens, like phthalates, bisfenols and pesticides may reduce the immune defense, thus increasing the risk of developing a severe COVID-19, as shown in a recent analysis performed by Wu et al. [124]. Endocrine disruptors interfere with the metabolism of sex steroids with increase of circulating androgens, a conditions that correlates with severity of lung involvement [104].

Xenoestrogens can be found in makeup, hair dyes, soaps, and perfumes, which are more commonly used by women. Despite the above, the impact of these compounds is still under investigation, particularly for their potential role in the increased prevalence of autoimmune diseases in women.

\subsection{Sex hormones blockers}

Estrogen deprivation and aromatase inhibitors therapy is a standard of treatment in estrogen receptor-positive mammary cancer. Aromatase inhibitors, such as anastrazole, letrozole and exemestane, inhibits the conversion of androgens to estrogens and accumulation of androgens could potentially lead to increases in TMPRSS2 gene expression. On the other side tamoxifen with its antiestrogenic, antiandrogenic and antiviral combined functions could be an ally in the fight to COVID-19 for patients with mammary cancer. Unfortunately, tamoxifen treatment is associated with an increased risk thromboembolism. In a recent study, SERMS appear as the most promising drugs in reducing SARS-CoV-2 infection in women affected by hormone-driven cancers when compared to other antiestrogenic therapies [125, 126]. More efforts will help a future understanding of the molecular basis of this putative protective effect, before the use of SERMs and/or other antiestrogenic therapies in the prevention of COVID-19. What we intend to recommend is that any decision in treating the patients with these drugs should be 


\begin{tabular}{|l|}
\hline Estrogen \\
Protect from CVD (control of endothelial function, induction of ACE2 \\
gene expression, modulation of insulin secretion) \\
Promote fetal lung maturation and function (decrease NLRP3 \\
expression, increase airway branching and surfactant production) \\
Reduce virus entry into the host cells (decrease TMPRSS2 gene \\
expression, increase ADAM-17 gene expression) \\
Modulate the immune response (activation of T cell response) \\
\hline Progesterone \\
Immunimodulatory functions \\
\hline Androgen \\
Promote fetal lung maturation (promotion of airway branching) \\
Increase virus entry into the host cells (increase in TMPRSS2 gene \\
expression) \\
Modulate the immune response (induction of cytokines production)
\end{tabular}

Fig. 1 Role of sex hormones in COVID-19

taken patient by patient, keeping in mind the accumulated knowledge.

Androgen deprivation and antiandrogen therapy is used routinely in prostate and in mammary cancer [127].

These treatments should not be discontinued for the fear of COVID-19 infection or complications.

Undergoing clinical studies will help to uncover positive or negative effects of sex hormones blockers in the spreading or progression of COVID-19, opening to the extended use of these drugs to both sexes and even to healthy individuals at increased risk of infection (Table 1).

\section{Conclusions}

In the handling of COVID-19 infection sex-related differences are represented in the initial approach to viral infections, in dimorphic hormonal signaling pathways, and in differential risk profile based on socio-cultural factors. Due to differences between different sexes in laboratory values and biomarkers, sex-dependent diagnosis and treatment methods may be needed for accurate and effective therapy.

Understanding the metabolic and endocrine dysfunctions potentially linked to the exacerbation of the SARS-CoV-2 infection may support the discovery and biological interpretation of unrecognized pathogenetic bases in COVID-19.

The sex differences observed in COVID-19 vulnerability emphasize the need to better understand the impact of sex on morbidity and lethality of the disease and to tailor treatment according to sex. The ongoing clinical studies for prevention and treatment of COVID-19 disease should certainly encompass sex-sensitive analyses. Targeted approaches that consider both sex and gender are urgently needed in the response efforts against COVID-19.

The accumulated data so far indicate that sex hormones could be critical in explaining sex-related differences in COVID-19 morbidity and mortality (Fig. 1). However, variables are several: normal, hypofunctional or hyperfunctional phenotypes could act differently, the genetic and epigenetic background is certainly playing a crucial role,steroidal sex hormones are not the same, and several synthetic and natural molecules interact with the steroid receptors.

Considering the clinical and therapeutic use of sex steroid hormones and/or their inhibitors is a possibility. Suddenly, estrogen and androgen receptor inhibitors appear as unexpected allies in the fight against COVID-19. The prospected potential implications of sex hormones in the morbidity and mortality of COVID-19 point to the importance that future epidemiological and clinical studies will be focused on the evaluation of hormonal therapy and hormonal deprivation in male and female patients.

Overall, the impact of biological sex is an essential consideration in the study and reporting of COVID-19 outcomes and clinical management.

Future policies should treasure this worldwide experience and governments will have to address the gendered impacts of the outbreak, the necessary step forward in the creation of equitable policies.

Acknowledgements This manuscript was supported by F.I.R.M.O. Foundation.

\section{Declarations}

Ethics Approval N.A.

Informed Consent N.A.

Conflict of Interest No conflict of interest.

\section{References}

1. Brandi ML, Giustina A. Sexual dimorphism of coronavirus 19 morbidity and lethality. Trends Endocrinol Metab. 2020;31:918-27.

2. Blair ML. Sex-based differences in physiology: What should we teach in the medical curriculum? Adv Physiol Educ. 2007;31:23-5.

3. Obese AC, Kim SC, Yin KJ, Lee JP, Hamblin MH. Sex differences in vascular physiology and pathophysiology: Estrogen and androgen signaling in health and disease. Am J Physiol Heart Circolo Physiol. 2017;313:H524-45.

4. Maeng LY, Milad MR. Sex differences in anxiety disorders: Interactions between fear, stress, and gonadal hormones. Horm Behav. 2015;76:106-17.

5. Fathi A, Addo MM, Dahlke C. Sex differences in immunity: Implications for the development of novel vaccines against 
emerging pathogens. Front Immunol. 2021;8(11): 601170. https://doi.org/10.3389/FIun.2020.601170.

6. Kinkead R, Schlenker E. Sex-based differences in respiratory control: Progress in basic physiology and clinical research. Respir Physiol Neurobiol. 2017;245:1-3. https://doi.org/10. 1016/j.resp.2017.08.013.

7. Horwath O, Moberg M, Larsen FJ, et al. Influence of sex and fiber type on the satellite cell pool in human skeletal muscle. Scand J Med Sci Sports. 2021;31(2):303-12. https://doi.org/10. 1111/sms.13848. ePub 20 Oct 2020.

8. Ambhore NS, Kalidhindi RSR, Sathish V. Sex-steroid signaling in lung diseases and inflammation. Adv Exp Med Biol. 2021;1303:243-73.

9. Archival B, Leahy MG, Kipp S, Sheel AW. An integrative approach to the pulmonary physiology of exercise: When biological sera matter? Eur J Appl Physiol. 2021 Apr 27. https://doi. org/10.1007/s00421-021-04690-9. Online ahead of print.

10. Rahimi G, Rahimi B, Panahi M, et al. An overview of Betacoronaviruses-associated severe respiratory syndromes, focusing on sex-type-specific immune responses. Int Immunopharmacol. 2021;92: 107365. https://doi.org/10.1016/j.intimp.2021.107365.

11. Stubbs DW, Krebs E, Bari M, et al. Sex differences in pain and pain-related disability among primary care patients with chronic musculoskeletal pain. Pain Med. 2010;11:232-9.

12. Tower J. Sex-specific gene expression and life span regulation. Trends Endocrinol Metab. 2017;28:735-47.

13. Compston JE, McClung MR, Leslie WD. Osteoporosis. Lancet. 2019;393:364-76.

14. Dale E, Davis M, Faustman D. A central role for transcription factor NF-kB in autoimmunity, genes, sex, and the immune system. Adv Physiol Educ. 2006;30:152-8.

15. Fagan TF, Faustman DL. Sex differences in autoimmunity. Adv Mol Cell Biol. 2004;34:295-306.

16. Huxley V. Sex and the cardiovascular system: The intriguing tale of how women and men regulate cardiovascular function differently. Adv Physiol Educ. 2007;31:17-22.

17. Bambra C, Albani V, Franklin P. COVID-19 and the gender health paradox. Scand J Pub Health. 2021;49:17-26.

18. Viswanathan M, Reddy S, Bergman N, et al. Screening to prevent osteoporosis fractures: Updated evidence report and systematic review from the US preventive services task force. JAMA. 2018;319:2532-51.

19. Yu Y, Chen J, Li D, et al. Systematic analysis of adverse event reports for sex differences in adverse drog events. Sci Rep. 2016;6:24955. https://doi.org/10.1038/srep24955.

20. Ruzzo A, Graziano F, Galli F, et al. Sex-related differences in impact on safety of pharmacogenetic profile for colon cancer patients treated with FOLFOX-4 or XELOX adjuvant chemotherapy. Sci Rep. 2019;9:11527. https://doi.org/10.1038/ s41598-019-47627-1.

21. Sha J, Qie G, Yao Q, et al. Sex differences on clinical characteristics, severity, and mortality in adult patients with COVID-19: A multicentre retrospective study. Front Med. 2021;8: 607059. https://doi.org/10.3389/famed.2021.607059.

22. Penna C, Mercurio V, Tocchetti CG, Pagliaro P. Sexrelated differences in COVID19 lethality. Br J Pharmacolk. 2020; $177: 4375-85$.

23. Okpechi SC, Fong JT, Gill SS, et al. Global sex disparity on CVID-19: A descriptive review of sex hormones and consideration for the potential therapeutic use of hormone replacement therapy in older adults. Aging Dis. 2021;12:671-83.

24. Ghebard C, Regista-Zagrosek V, Neuhauser HK, Morgan R, Klein SL. Impact of sex and gender on COVID-19 outcomes in Europe. Biol Sex Differ. 2020;11:29.

25. Nicholls JM, Poon LL, Lee KC, et al. Lung pathology of fatal severe acute respiratory syndrome. Lancet. 2003;361:1773-8.
26. Karlberg J, Chong DS, Lai WY. Do men have a higher case fatality rate of severe acute respiratory syndrome than women do? Am J Epidemiol. 2004;159:229-31.

27. Channappanavar R, Fett C, Mack M, et al. Sex-based differences in susceptibility to severe acute respiratory syndrome coronavirus infection. J Immunol. 2017;198:4046-53.

28. Matsuyama R, Nishiura H, Kutsuma S, Hayakawa K, Ohmagari N. Clinical determinants of the severity of Middle East respiratory syndrome (MERS): A systematic review and meta-analysis. BMC Public Health. 2016;16:1203.

29. Klein SL, Flanagan KL. Sex differences in immune responses. Nat Rev Immunol. 2016;16:626-38.

30. Viboud C, Eisenstein J, Reid AH, et al. Age- and sex-specific mortality associated with the 1918-1919 influenza pandemic in Kentucky. J Infect Dis. 2013;207:721-9.

31. van Zyl-Smith RN, Richards G, Leone FT. Tobacco smoking and COVID-19 infection. Lancet Respir Med. 2020;8:664-5.

32. Snell DM, Turner JMA. Sex chromosome effects on malefemale differences in mammals. Curr Biol. 2018;28:R1313-24.

33. Schurz H, Salie M, Tromp G, et al. The X chromosome and sex-specific effects in infectious disease susceptibility. Hum Genomics. 2019;13:12.

34. Santos RA, e Silva ACS, Maria C, et al. Angiotensin (1-7) is an endogenous ligand for the G-protein coupled receptor Mas. PNAS. 2003;100:8258-63.

35. Gomez J, Abaiceta GM, Garcia-Clemente M, et al. Angiotensinconverting enzymes (ACE, ACE2) gene variants and COVID19 outcome. Gene. 2020;762: 145102. https://doi.org/10.1016/j. gene.2020.145102.

36. Xie X, Chen J, Wang X, Zhang F, Liu Y. Age- and gender-related difference in ACE2 expression in rat lung. Life Sci. 2006;78:2166-71.

37. Zheng YY, Ma YT, Zhang YJ, Xie X. COVID-19 and the cardiovascular system. Nat Rev Cardiol. 2020;17(5):259-60.

38. Devaux CA, Romani JM, Raoult D. ACE2 receptor polymorphism: Susceptibility to SARS-CoV-2, hypertension, multiorgan failure, and COVID-19 disease outcome. J Microbiol Immunol Infect. 2020;53:425-35.

39. Sabharwal N, Sharifi N. HSD3B1 genotype conferring adrenalrestrictive and adrenal-permissive phenotype in prostate cancer and beyond. Endocrinology. 2019;160:2180-8.

40. FitzGerald LM, Agalliu I, Johnson K, et al. Association of TMPRSS2ERG gene fusion with clinical characteristics and outcomes: Results from a population-based study of prostate cancer. BMC Cancer. 2008;8:230. https://doi.org/10.1186/1471-2407-8-230.

41. Asselta R, Paraboschi EM, Mantovani A, Dugs S. ACE2 and TMPRSS2 variants and expression as candidates to sex and country differences in COVID-19 severity in Italy. medRxiv. 2020;12:10097-8.

42. Selek A, Guclu M, Bolu SE. COVID-19 pandemic: What about the gonads? Hormones. 2021;20:259-68. https://doi.org/10. 1007/s42000-021-00277-3.

43. Pario-Castineira E, Clohisey S, Klaric L, et al. Genetic mechanisms of critical illness in COVID-19. Nature. 2021;591:92-8.

44. Klein SL, Hodgson A, Robinson DP. Mechanisms of sex disparities in influenza pathogenesis. J Leukocyte Biol. 2012;92:67-73.

45. Belmonte RL, Corbally M-K, Duneau DF, Regan JC. Sexual dimorphisms in innate immunity and responses to infection in Drosophila melanogaster. Front Immunol. 2020;10:3075-93.

46. Jin J-M. Gender differences in patients with COVID-19: Focus on severity and mortality. Front Pub Health 2020;8:152.

47. Jensen-Fangel S, Mohey R, Johnson SP, et al. Gender differences in hospitalization rates for respiratory tract infections in Danish youth. Scand J Infect Dis. 2004;36:31-6.

48. Bain CC, Gibson DA, Steers NJ, et al. Rate of replenishment and micro environment contribute to the sexually dimorphic 
phenotype and function of peritoneal macrophages. Sci Immunol. 2020;5:eabc4466. https://doi.org/10.1126/sciimmunol.abc4466.

49. Vasanthakumar A, Chisanga D, Blume J, et al. A sex-specific adipose tissue imprinting of regulatory T cells. Nature. 2020;579:581-5.

50. Marquez EJ, Chung C, Marches R, et al. Sexual-dimorphism in human immune system aging. Nat Commun. 2020;11:751. https://doi.org/10.1038/s41467-020-14396-9.

51. Gal-Oz ST, Maier B, Yoshida H, et al. ImmGen report sexual dimorphism in the immune system transcrptome. Nat Commun. 2019;10:4295. https://doi.org/10.1038/541467-019-12348-6.

52. Ruan Q, Yang K, Wang W, Jiang L, Song J. Clinical predictors of mortality due to COVID-19 based on an analysis of data of 158 . Patients from Wuhan, China. Intensive Care Med. 2020;46:846-8.

53. Shi Y, Wang Y, Shao C, et al. COVID-19 infection: The perspectives on immune responses. Cell Death Differ. 2020;27:1451-4.

54. Bienvenue LA, Noonan J, Wang X, Peter K. Higher mortality of COVID-19 in males: Sex differences in immune response and cardiovascular comorbidities. Cardiovasc Res. 2020;0:1-10.

55. Takahashi T, Ellingson MK, Wang P, et al. Sex differences in immune responses that underlie COVID-19 disease outcomes. Nature. 2020;588:315-20.

56. Guzik TJ, Mohidfin SA, Dimarco A, et al. COVID-19 and the cardiovascular system: Implications for risk assessment, diagnosis, and treatment options. Cardiovasc Res. 2020;116:1666-87.

57. Penna C, Mercurio V, Tocchetti CG, Pagliaro P. Sexrelated differences in COVID-19 lethality. Br J Pharmacol. 2020;177:4375-85.

58. Moccia F, Gerbino A, Lionetti V, et al. COVID-19-associated cardiovascular morbidity in older adults: A position paper from the Italian Society of Cardiovascular Researches. Geoscience. 2020;42:1021-49.

59. Mathew D, Jiles JR, Baxter AE, et al. Deep immune profiling of COVID-19 patients reveals patient heterogeneity and distinct immunophenotypes with implications for therapeutic interventions. Science. 2020;369:eabc8511. https://doi.org/10.1126/science.abc8511.

60. Sattar N, McInnes IB, McMurray YJV. Obesity is a risk factor for severe COVID-19 infection: Multiple potential mechanisms. Circulation. 2020;142:4-6.

61. Palmer BF, Clegg DJ. The sexual dimorphism of obesity. Mol Cell Endocrinol. 2015;402:113-9.

62. Lim S, Bae JH, Kwon H-S, Nhuck MA. COVID-19 and diabetes mellitus: From pathophysiology to clinical management. NRE. 2021;17:11-30.

63. Diabetes and COVID_19-related mortality in women and men in the UK Biobank: Comparisons with influenza/pneumonia and coronary heart disease. Diab Care. 2021;44:e22-4.

64. Kautzky-Willer A, Harreiter J, Pacini G. Sex and gender differences in risk, pathophysiology and complications in type 2 diabetes mellitus. Endocrinol Rev. 2016;37:278-316.

65. Peters SA, Woodward M. Sex differences in the burden and complications of diabetes. Curr Diab Rep. 2018;18:33.

66. Tramunt B, Smati S, Grandgeorge N, et al. Sex differences in metabolic regulation and diabetes susceptibility. Diabetologia. 2020;63:453-61.

67. Becklake MR, Kauffmann F. Gender differences in airway behavior over the human life span. Thorax. 1999;54:1119-38.

68. Today JS, Nielsen HC. The sex difference in fetal lung surfactant production. Exp Lung Res. 1987;12:1-19.

69. Molgat-Seon Y, Peters CM, Sheel AW. Sex-differences in the human respiratory system and their impact on resting pulmonary function and the integrative response to exercise. Curr Opin Physiol. 2018;6:21-7.

70. Langhammer A, Johnson R, Gulsvik A, Holmes TL, Bjemer L. Sex differences in lung vulnerability to tobacco smoking. Eur Resp J. 2003;21:1017-23.
71. Di Filippo L, Formenti AM, Doga M, et al. Radiological thoracic vertebral fractures are highly prevalent in CIVID-19 and predict disease outcomes. J Clin Endocrinol Metabolism. 2021;106:e602-14. https://doi.org/10.1210/clines/dgaa738.

72. Pandit P, Perez RL, Roman J. Sex-based differences in interstitial lung disease. Am J Med Sci. 2020;360:467-73.

73. Pivonello R, Auriemma RS, Pivonello C, et al. Sex disparities in COVID-19 severity and outcome: Are men weaker or women stronger? Neuroendocrinology. 2020;11:53.

74. Wu FC, Tamar A, Beynin JM, et al. EMAS Group. Identification of late-onset hypogonadism in middle-aged and elderly men. $\mathrm{N}$ Engl J Med. 2010;363:123-35.

75. Duga S, Asselta R, Lazzerini M, et al. Impact of anti-androgenic therapies on COVID-19: An observational study in male population from a COVID-19 regional center in Lombardy (Italy). medRxiv. 2020;2020.04.20.20068056.

76. McCoy J, Gordon A, Cadegiani FA, et al. Proxalutamide reduces the rate of hospitalization for COVID-19 male outpatients: A randomized double-blinded placebo-controlled trial. Front Med. 2021;8: 668698. https://doi.org/10.3389/famed.2021.668698.

77. Montopoli M, Zumerle S, Vettor R, et al. Androgen- deprivation therapies for prostate cancer and risk of infection by SARS-CoV-2: A population-based study (N=4532). Ann Oncol. 2020;31:1040-5.

78. Liang W, Guan W, Chen R, et al. Cancer patients in SARSCoV-2 infection: A nationwide analysis in China. Lancet Oncol. 2020;21:335-7.

79. Comas C, Carreras E. COVID-19 and pregnancy: An opportunity to correct an historic gender bias. J Med Virol. 2021;93:22-4.

80. Mauvais-Jarvis F, Klein SL, Levin ER. Estradiol, progesterone, immunomodulation, and COVID-19 outcomes. Endocrinology. 2020;161:1-8.

81. Honorato-Sampao K, Pereira VM, Santos RA, Reis AM. Evidence that angiotensin(1-7) is an intermediate of gonadotrophininduced oocyte maturation in the rat preovulatory follicle. Exp Physiol. 2012;97:642-50.

82. Garg R, Agrawal P, Gautam A, et al. COVID-19 outcomes in postmenopausal and perimenopausal females: Is estrogen hormone attributing to gender differences? J Midlife Health. 2021;11:250-6.

83. Fait T. Menopause hormone therapy: Latest developments and clinical practice. Drugs Context. 2019;8:212551.

84. Park KU, Gregory M, Bazan J, et al. Neoadjuvant endocrine therapy use in early stage breast cancer during the covid-19 pandemic. Breast Cancer Res Treat. 2021;188:249-58.

85. Hortsman AM, Dillon EL, Urban RJ, Sheffield-Moore M. The role of androgens and estrogens on healthy aging and longevity. J Gerontol Series A: Biomed Sci Med Sci. 2012;67:1140-52.

86. Xu H, Wang Z, Feng C, et al. Effects of SARS-CoV-2 infection on male sex-related hormones in recovering patients. Andrology. 2021;9:107-14.

87. Porter V, Greendale G, Schocken M, Zhou X, Effros R. Immune effects of hormone replacement therapy in post-menopausal women. Exp Gerontol. 2001;36:311-26.

88. Taneja V. Sex hormones determine immune response. Front Immunol. 2018;9:1931.

89. Erfinanda L, Ravindran K, Kohse F, et al. Oestrogen-mediated upregulation of the Mas receptor contributes to sex differences in acute lung injury and lung vascular barrier regulation. Eur Resp J. 2021;57:2000921.

90. Mompeon A, Lazaro-Franco M, Bueno-Beti C, et al. Estradiol, acting through ERalpha, induces endothelial non-classic reninangiotensin system increasing angiotensin 1-7 production. Mol Cell Endocrinol. 2016;422:1-8.

91. Baran-Gale I, Purvis JE, Sethupathy P. An integrative trascriptomics approach identifies MiR-503 as a candidate master regulator of the estrogen response in the MCF-7 breast cancer cells. RNA. 2016;22:1592-603. 
92. Ragia G, Manolopoulos VG. Assessing COVID-19 susceptibility through analysis of the genetic and epigenetic diversity of ACE2mediated SARS-CoV-2 entry. Pharmacogenomics. 2020;21:1311-29.

93. Cheng $\mathrm{C}, \mathrm{Wu} \mathrm{H}$, Wang $\mathrm{M}$, et al. Estrogen ameliorates allergic airway inflammation by regulating activation of NLRP3 in mice. Biosci Rep. 2019;39. https://doi.org/10.1042/BSR20181117.

94. Lemes RMR, Costa AJ, Bartolomeo CS, et al. 17beta-estradiol reduces SARS-CoV-2 infection in vitro. Physiol Rep. 2021;9: e14707. https://doi.org/10.14814/phy2.14707.

95. ClinicalTrials.gov. Estrogen patch for COVID-19 symptoms. Sponsor: Sharon Nachman, Stony Brook University, Identifier: NCT04359329.

96. Balanger A, Candas B, DuPont A, et al. Changes in serum concentrations of conjugated and unconjugated steroids in 40- to 80-year-old men. J Clin Endocrinol Metab. 1994;79:1086-90.

97. Douglas GC, O'Bryan MK, Hedger MP, et al. The novel angiotensin-converting enzyme (ACE) homologous, ACE2, is selectively expressed in adult Leydig cells of the testis. Endocrinology. 2004;145:4703-11.

98. Maggio M, Basaria S, Ceda GP, et al. The relationship between testosterone and molecular markers of inflammation in older men. J Endocrinol Invest. 2005;28:116-9.

99. Townsend EA, Miller VM, Prakash YS. Sex differences and sex steroids in lung health and disease. Endocrinol Rev. 2012;33:1-47.

100. Mikkonen L, et al. Androgen receptor and androgen-dependent gene expression in lung. Mol Cell Endocrinol. 2010;317:14-24.

101. Pozzilli P, Lenzi A. Testosterone, a key hormone in the context of COVID-19 pandemic. Metab Clin Exp. 2020;108:154252.

102. Hoffmann M, Kleine-Weber H, Schroeder S, et al. SARS-CoV-2 cell entry depends on ACE2 and TMPRSS2 and is blocked by a clinically proven protease inhibitor. Cell. 2020;181:271-80.

103. Barnes BJ, Adrover JM, Baxter-Stoltzfus A, et al. Targeting poential drivers of COVID-19: Neutrophil extra cellular traps. J Exp Med. 2020;217:e20200652.

104. Wambier CG, Goren A. SARS-CoV-2 infection is likely to be androgen-mediated. J Am Acad Dermatol. 2020;83:308-9.

105. Leach DA, Mohr A, Giotis ES, et al. The antiandrogen enzolutamide diwnregulates TMPRSS2 and reduces cellular entry of SARS-CoV-2 in human lung cells. Nature Comm. 2021;12:4068.

106. Bravaccini S, Fonzi E, Tebaldi M, et al. Estrogen and androgen receptor inhibitors: Unexpected allies in the fight against COVID-19. Cell Transplant. 2021;30:1-6.

107. Montopoli M, Zumerle S, Vettor R, et al. ANdrogen-dependent therapies for prostate cancer and risk of infection by SARSCoV-2: A population-based study $(\mathrm{N}=4532)$. Ann Oncol. 2020;31:1040-5.

108. Reis FM, Bouissou DR, Pereira VM, et al. Angiotensin-(1-7), its receptor Mas, and the angiotensin-converting enzyme type 2 are expressed in the human ovary. Fertil Steril. 2011;95:176-81.

109. Vas-Silva J, Carneiro MM, Ferreira MC, et al. The vasoactive peptide angiotensin-(1-7), its receptor Mas, and the angiotensinconverting enzyme type 2 are expressed in the human endometrium. Reprod Sci. 2009;16:247-56.

110. Wang $\mathrm{Z}, \mathrm{Xu} \mathrm{X}$. scRNA-seq profiling of human testis reveals the presence of the ACE2 receptor, a target for SARS-CoV-2 infection in spermatogonia, Leydig and Sertoli cells. Cells. 2020;9. https://doi.org/10.3390/cells9040920.

111. Illiano E, Trama F, Costantini E. Could COVID-19 have an impact on make fertility? Andrologia. 2020;52: e13654. https:// doi.org/10.1111/and.13654.
112. Seeland U, Coluzzi F, Simmaco M, et al. Evidence for treatment with estradiol for women with SARS-CoV-2 infection. BMC Med. 2020;18:369. https://doi.org/10.1186/s12916-020-01851-z.

113. Managing thromboembolic risk with menopausal hormone therapy and hormonal contraception in the COVID-19 pandemic. Recommendations from the Spanish Menopause Society. Maturitas. 2020;137:57-62.

114. Suba Z. Prevention and therapy of COVID-19 via exogenous estrogen treatment for both male and female patients: An opinion paper. J Pharm Pharm Sci. 2020;23:75-85.

115. Darabi P, Khazali H, Natanzi MM. Therapeutic potentials of the natural plant flavonoid apigenin in polycystic ovary syndrome in rat model: Via modulation of pro-inflammatory cytokines and antioxidant activity. Gynecol Endocrinol. 2019;30:1-6.

116. Meng Z-J, Wang C, Meng L-T, et al. Sodium tanshinone IIA sulfonate attenuated cardiac dysfunction and improves survival of rats with cecal ligation and puncture-induced sepsis. J Nat Med. 2018;72:846-55.

117. Elfiky AA. Natural products may interfere with SARS-CoV-2 attachment to the host cell. J Biomolecular Struct Dyn. 2021;39:3194-203.

118. Koramouzis MV, Papavassiliou KA, Adamopoulos C, Papavassiliou AG. Targeting androgen/estrogen receptors crosstalk in cancer. Trends Cancer. 2016;2:35-48.

119. Srivatsan SR, McFaline-Figueroa JL, Ramani V, et al. Massively multiplex chemical transcriptomics at single-cell resolution. Science. 2020;367:45-51.

120. Altan N, Chen Y, Schindler M, Simon SM. Tamoxifen inhibits acidification in cells independent of the estrogen receptor. Proc Nat Acad Sci USA. 1999;96:4432-7.

121. Brabek J, Rosel D, Smetana K, Fernandez M. Repurposing of bazedoxifene to prevent cytokine storm in COVID-19 patients. Science. 2020;368:473-4.

122. Li H, Xiao H, Lin L, et al. Drug design targeting protein-protein interactions (PPIs) using multiple ligands simultaneous docking (MLSD) and drug repositioning: Discovery of raloxifene and bazedoxifene as novel inhibitors of IL-6/GP130 interface. J Med Chem. 2014;57:632-41.

123. Jeon S, Ko M, Lee J, et al. Identification of antiviral drug candidates against SARS-CoV-2 from FDA approved drugs. Antimicrob Agents Chemother. 2020;64:e00819-e820.

124. Wu Q, Coumoul X, Grandjean P, et al. Endocrine disrupting chemicals and COVID-19 relationship: A computational systems biology approach. Environ Int. 2020;30: 106232. https://doi.org/ 10.1016/j.ending.2020.106232.

125. Ortona E, Pierdominici M, Maselli A, et al. Sex-based differences in autoimmune diseases. Ann Ist Super Sanità. 2016;52:205-12.

126. Montopoli M, Zorzi M, Cocetta V, et al. Clinical outcome of SARS-CoV-2 infection in breast and ovarian cancer patients who underwent antiestrogenic therapy. Ann Oncol. 2021;32:676-7.

127. Student S, Hejmo T, Poterala-Hejmo A, et al. Can antiandrogenic drugs used fir the treatment of prostate cancer reduce SARSCoV-2 infection? Eur J Pharm. 2020;866:172783.

Publisher's Note Springer Nature remains neutral with regard to jurisdictional claims in published maps and institutional affiliations. 